\title{
Exclusión Social e Inequidad en Salud en México: Un Análisis Socio-espacial
}

\author{
A socio-spatial analysis of social exclusion and inequity \\ in health in Mexico
}

Guillermo J. González-Pérez¹, María G. Vega-López¹, Samuel Romero-Valle, Agustín Vega-López ${ }^{1}$ y Carlos E. Cabrera-Pivaral ${ }^{1,2}$

1 Centro Universitario de Ciencias de la Salud, Universidad de Guadalajara, México. ggonzal@ cencar.udg.mx, magvega@cucs.udg.mx, s_romerovalle@yahoo.com.mx, agustin_vegalopez@yahoo.com.mx

2 Instituto Mexicano del Seguro Social, Delegación Jalisco, México. carlos_cabrera@prodigy.net.mx

Recibido 8 Enero 2008/Enviado para Modificación 2 Mayo 2008/Aceptado 8 Septiembre 2008

\section{RESUMEN}

Objetivo Determinar la relación existente entre exclusión social e inequidad en salud a nivel estatal y municipal en México en años recientes.

Métodos Se calcularon tasas estandarizadas de mortalidad relacionadas con enfermedades trasmisibles en la infancia, el embarazo y el parto así como producto de causas que podrían considerarse potencialmente evitables; estas tasas se calcularon por estados, por estados agrupados en cuartiles según nivel de marginación y por municipios agrupados según grado de marginación. Para medir la inequidad, se utilizaron indicadores como la razón de tasa, el coeficiente de Gini y el índice de inequidades en salud (INIQUIS)

Resultados Se observa un claro exceso de mortalidad en los Estados agrupados en el Cuartil IV (mayor marginación) en relación con el Cuartil I (menor marginación) y a la inversa, los recursos y servicios disponibles en el Cuartil I son mayores que los existentes en el Cuartil IV. El Coeficiente de Gini alcanza su valor más alto en la tasa de mortalidad por anemias nutricionales $(0,44)$. El exceso de mortalidad es evidente en los municipios considerados como de muy alta marginación; el INIQUIS más elevado se observa en los Estados ubicados en el Cuartil IV, sobre todo Chiapas, Oaxaca y Guerrero.

Conclusiones Existe en México una notoria inequidad en salud, asociada a los altos niveles de exclusión social; para modificar esta situación se necesitan profundos cambios estructurales que impulsen el desarrollo social, y permitan reducir las desventajas injustas a las que están expuestos importantes núcleos poblacionales del país.

Palabras Clave: Inequidad en salud, marginación, mortalidad, México (fuente: DeCS, BIREME). 


\begin{abstract}
Objective Determining the relationship between social exclusion and health inequity at state and municipal level in Mexico during recent years.

Methods Adjusted mortality rates were calculated for 2005 (related to transmissible illnesses in childhood, pregnancy, childbirth and being produced by causes considered potentially avoidable); rates were calculated by states, for states grouped in quartiles according to marginalisation level and for municipalities grouped according to degree of marginalisation. Indicators such as rate ratio, Gini coefficient and the inequities in health index $(\mathrm{IHI})$ were used for measuring such inequity,

Results A clear excess of mortality was observed in the states grouped in the $4^{\text {th }}$ quartile (highest marginalisation) in relationship to the $1^{\text {st }}$ quartile (lowest marginalisation); conversely, resources and health services in the $1^{\text {st }}$ quartile were evidently higher than those in the $4^{\text {th }}$ quartile. The Gini coefficient reached its highest value in the mortality rate for nutritional anaemia (0.44). Excess mortality was evident in those municipalities considered as having very high marginalisation; the highest $\mathrm{IHI}$ was observed in the states located in the $4^{\text {th }}$ quartile (Chiapas, Oaxaca and Guerrero) when analysing mortality related to childhood, pregnancy, childbirth and potentially avoidable mortality.

Conclusions Notorious health inequality exists in Mexico, associated with high prevalent levels of social exclusion in different areas of the country. Deep structural changes are needed to modify this situation, promote social development and lead to reducing the unfair disadvantages to which important population groups are exposed.
\end{abstract}

Key Words: Health inequity, marginalisation, mortality, Mexico (source: MeSH, NLM).

mérica Latina ha tenido avances considerables en el campo de la salud en
años recientes, reflejados en el incremento de la esperanza de vida al
nacer y la reducción de las tasa de mortalidad infantil en la mayoría de los países de la región; no obstante, existen todavía evidentes disparidades en los logros alcanzados en este campo por los distintos países, e igualmente, grandes diferencias al interior de cada uno de ellos, en indicadores como los mencionados previamente, o la mortalidad materna, por citar solo algunos; estas disparidades parecen responder -en buena medida- a las notables desigualdades socioeconómicas que subyacen en la región; diversos autores han coincidido en señalar a Latinoamérica como la región más desigual del mundo: mientras el decil más rico recibe el $48 \%$ del ingreso total, el decil más pobre recibe apenas el 1,6 \% del ingreso (1).

En este contexto, México no es la excepción: según la Encuesta Nacional de Ingresos y Gasto de los Hogares 2005, el 47 \% de los habitantes vivía en pobreza patrimonial, esto es, que su ingreso es menor al necesario para cubrir el 
patrón de consumo básico de alimentación, salud, educación, vestido, vivienda y transporte público; por otra parte el ingreso promedio en el decil de mayores ingresos era más de 30 veces superior al del decil de menores ingresos, que apenas recibe el 1,3 \% del total de los ingresos nacionales (2). Esta situación no ha sufrido cambios relevantes en las últimas décadas y, obviamente, también repercute en las condiciones de salud de la población mexicana (2).

Una forma de aproximación al análisis de las desigualdades sociales desde una perspectiva socio-espacial lo constituye, en el contexto mexicano, el análisis de la marginación. El Consejo Nacional de Población (CONAPO) define a la marginación como un fenómeno estructural múltiple que toma en cuenta dimensiones (Educación, Vivienda, Ingresos Monetarios y la Distribución de la población), formas de exclusión y la intensidad de la exclusión en el proceso de desarrollo y el disfrute de sus beneficios (por ejemplo analfabetismo, hacinamiento, población ocupada, población rural; en total, 10 indicadores de exclusión) (3). Así, el índice de marginación calculado por CONAPO tanto a nivel estatal como municipal mide la intensidad global de la marginación socioeconómica en un área determinada, y por consiguiente, refleja en buena medida las condiciones de pobreza -y sobre todo- de exclusión social de cada área.

Si bien la marginación -en tanto se deriva de una condición de pobrezaimplica estar al margen de un conjunto de opciones sociales, el concepto de exclusión social lleva implícito el hecho de quedar fuera de la dinámica social, de estar excluido de la participación en la toma de decisiones, en la creación y el disfrute de bienes y servicios, y es expresión y resultado de una determinada estructura social (4). Así, los indicadores calculados por CONAPO revelan que en numerosas áreas geográficas de México prevalecen altos niveles de marginación, pero también de exclusión social que impactan en la salud de la población.

La relación entre condiciones socioeconómicas y estado de salud de la población ha sido ampliamente documentada; en particular, el interés por analizar las disparidades en salud entre grupos sociales como resultado de sus diferentes condiciones de vida parece remontarse, como señala Starfield (5), al conocido estudio de Engels "La situación de la clase obrera en Inglaterra" (6), desarrollado en la primera mitad del siglo XIX. Algunas de las ideas subyacentes en el análisis de Engels -“...¿cómo es posible que la clase pobre pueda disfrutar de buena salud y vivir mucho tiempo? ¿Qué otra cosa puede esperarse sino una enorme mortalidad, epidemias permanentes, y un debilitamiento progresivo e ineluctable de la generación de los trabajadores?...”- plantean la injusticia de una situación innecesaria 
y evitable si las condiciones de vida de este grupo social fueran sustancialmente mejores.

En fechas recientes, se ha definido la equidad en salud como "la ausencia de diferencias sistemáticas y potencialmente remediables en uno o más aspectos de la salud, la educación, la nutrición y las condiciones de vida de las personas en el contexto de poblaciones o grupos poblacionales definidos social, económica, demográfica y/o geográficamente” (7); desde esta perspectiva, parecería lógico esperar que la existencia de importantes áreas geográficas en México socialmente marginadas y con altos niveles de exclusión social se tradujera en la presencia de notorias inequidades sanitarias (8).

Este estudio pretende determinar la relación existente entre exclusión social e inequidad en salud en México en años recientes, analizando distintos indicadores de mortalidad -sobre todo aquella que pudiera ser considerada como potencialmente evitable- y de recursos desde una perspectiva socio-espacial, esto es, tomando en cuenta datos agregados a nivel estatal y municipal.

\section{METODOLOGÍA}

México es uno de los países más extensos y poblados de Latinoamérica, con un territorio de $1964375 \mathrm{~km}^{2}$, y algo más de 103 millones de habitantes en el año 2005 (9). Su Producto Interno Bruto per cápita fue de USD $\$ 7310$ (10), aunque una notable proporción de la población vive en condiciones de pobreza: desde alrededor del 20 \% según el Programa de Desarrollo de las Naciones Unidas (11) hasta el $47 \%$ de pobreza patrimonial antes mencionado (2). México está dividido administrativamente en 31 estados y un Distrito Federal, así como 2454 municipios, cuyo desarrollo económico y social varía notablemente.

La información sobre indicadores de recursos y servicios (gasto público en salud, médicos por habitante, partos con atención médica) y mortalidad utilizada en este estudio proviene de las bases de datos disponibles en el Sistema Nacional de Información en Salud (SINAIS) de la Secretaría de Salud (12); en especial, las bases de datos de mortalidad generadas por este organismo a partir de los datos recolectados por el Instituto Nacional de Estadísticas, Geografía e Informática (INEGI) permiten un estudio de esta índole dado su grado de desagregación por sexo, edad, causa, lugar de residencia y grado de marginación. Específicamente, se estudiaron para 2005 indicadores de la mortalidad relacionados con las enfermedades trasmisibles en la infancia, el embarazo y el parto (mortalidad infantil, mortalidad materna, mortalidad perinatal, y mortalidad en menores de 5 años por 
diarreas e infecciones respiratorias agudas) así como un conjunto de causas de mortalidad que podrían considerarse como potencialmente evitables, clasificadas de acuerdo con la X Clasificación Internacional de Enfermedades: Enfermedades Infecciosas Intestinales (A00-A09), Homicidios (X85-Y09), Tuberculosis pulmonar (A15-A16), Desnutrición (E40-E46), Anemias Nutricionales (D50-D53) (12). La población para el cálculo de tasas estatales para los años 2004 y 2005 se obtuvo de las Proyecciones de Población de CONAPO $(13,14)$. Las tasas se ajustaron por edad, considerándose como estándar la población de México en 2005.

Con la información por estados, se calcularon dos indicadores que permiten la jerarquización de problemas y necesidades de salud: por una parte, el coeficiente de Gini (una conocida medida de la desigualdad basada en la curva de Lorenz), que puede asumir valores que van desde 0 hasta 1 ; cuanto más se acerca a la unidad, el coeficiente indica mayor desigualdad; y por otra, el índice de inequidades en salud (INIQUIS), una medida que se construye mediante un procedimiento iterativo a partir de un conjunto de indicadores que reflejen el concepto de inequidad en salud, medidos en un grupo de unidades geográficas, en este caso los estados. La idea básica del procedimiento es definir un indicador inicial, representativo, que se entiende no debe dejar de formar parte del índice y, a partir de ahí, realizar una incorporación paso a paso de indicadores mediante una regla de decisión. Los valores de INIQUIS en cada unidad geográfica pueden oscilar entre 0 y 1 , y representan la distancia media a las unidades geográficas con mejor situación en términos de los indicadores que integran el índice, de modo que valores próximos a 1 indican una peor situación -en este caso una mayor inequidad- y viceversa, valores próximos a 0 corresponden a la mejor situación en términos de salud. Ambas medidas se calcularon mediante el programa EPIDAT, versión 3.1 (15).

Junto al análisis anterior, y a partir de ordenar a los 32 estados según su índice de marginación social en 2005 (16), se ha procedido en este estudio a agrupar los estados en cuartiles, incluyendo en el Cuartil I los 8 estados con menor grado de marginación y en el Cuartil IV, los 8 estados con mayor marginación; en tal sentido, se calcularon tasas de mortalidad -ajustadas por edad- por causas seleccionadas y cuartiles, para 2005 (tomándose como estándar la población de México en 2005) y se calculó el exceso de mortalidad del Cuartil IV con relación al Cuartil I mediante el empleo de la razón de tasas. 
Por último, se calcularon las tasas de mortalidad por causas seleccionadas según grado de marginación municipal para 2005 y la razón entre las tasas del grupo de municipios con muy alta marginación y el de muy baja marginación.

\section{RESULTADOS}

En la Figura 1 se puede apreciar la distribución de los Estados en cuartiles según su índice de marginación en 2005; así, en el Cuartil IV, donde se concentran los estados con mayor marginación social, se encuentran Guerrero, Chiapas, Oaxaca, Veracruz, Hidalgo, San Luís Potosí, Puebla y Campeche, siendo los tres primeros mencionados los que presentan el grado de marginación más elevado (con índices de marginación que oscilan entre 0,56 en Campeche y 2,41 en Guerrero). Este conjunto de estados -localizados mayormente hacia el sur del país- se caracteriza por tener una notable proporción de población rural (53,4\%) e indígena (15,4 \%), el menor producto interno bruto per cápita, y por concentrar, tanto en términos absolutos como relativos, la mayor población infantil y anciana del país (16).

Por su parte, en el Cuartil I se ubican estados como el Distrito Federal, Nuevo León, Baja California, Coahuila, Aguascalientes, Jalisco, Sonora y Colima, con el grado de marginación más bajo (que va de -0.74 en Colima a -1.50 en el Distrito Federal). Solo el 11 \% de la población de los estados ubicados en este cuartil vive en áreas rurales, y apenas el $1 \%$ habla alguna lengua indígena. Su producto interno bruto per cápita es el más elevado, (casi triplica el del Cuartil IV) y tiene la más baja proporción de población menor de 15 años (16).

En la Tabla 1, por su parte, se presentan indicadores de mortalidad y de recursos y servicios calculados por cuartil, para 2005. En lo referido a la mortalidad, los datos ponen de manifiesto un claro exceso de mortalidad en el Cuartil IV (mayor marginación) en relación con el Cuartil I (menor marginación), destacando que el riesgo de muerte por anemias nutricionales en el Cuartil IV quintuplica el del Cuartil I, y por enfermedades infecciosas intestinales, más que lo duplica.

Se puede observar también, en términos generales, que existe una tendencia al incremento de las tasas en tanto aumenta el grado de marginación quizá con la excepción del homicidio y la tuberculosis pulmonar- y que el coeficiente de Gini alcanza sus valores más altos en la tasa de mortalidad por anemias nutricionales y por tuberculosis pulmonar. 
Figura 1. México: Estados ordenados en cuartiles según índice de marginación Estatal, 2005

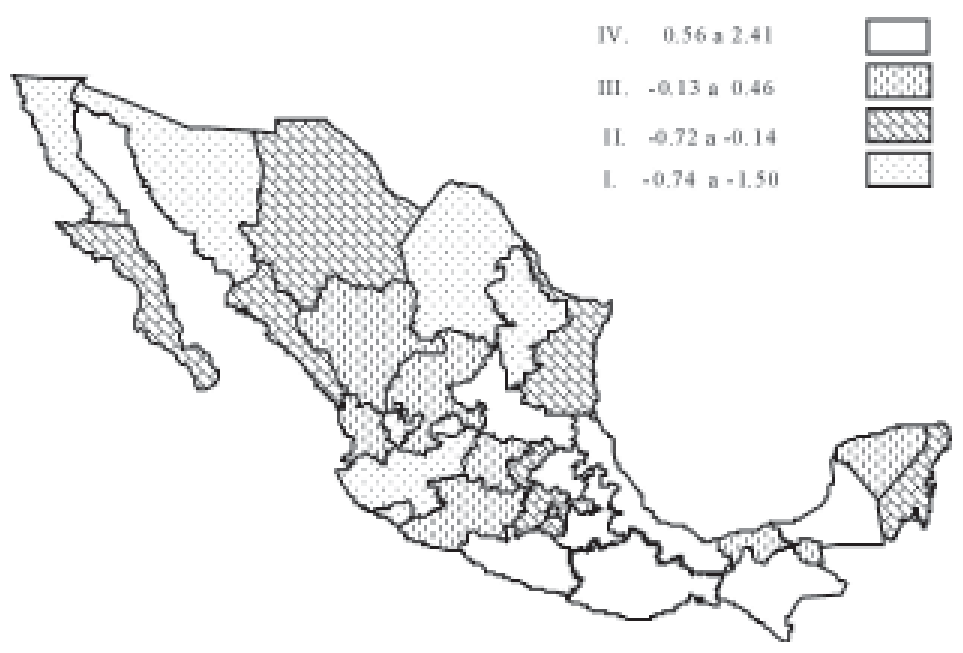

Fuente: Elaboración propia a partir de datos de CONAPO (2006)

En lo concerniente a los indicadores de recursos y servicios, se observa claramente como en el Cuartil I se dispone de mayores recursos: el gasto público en salud, y los indicadores de recursos humanos en dicho cuartil duplican lo observado en el Cuartil IV, donde también hay una proporción mucho más reducida de derechohabientes de las diversas instituciones de la seguridad social y un porcentaje menor de los partos son atendidos por personal especializado. En este caso, la tendencia también parece indicar que a mayor marginación menos recursos humanos especializados y menos recursos financieros. Los coeficientes de Gini son más elevados en indicadores como enfermeras y médicos por habitantes, y el gasto público en salud.

En la Tabla 2 se presentan las tasas de mortalidad según municipios agrupados por grado de marginación. Como puede apreciarse, en todos los casos las tasas calculadas para aquellos municipios en la categoría de muy alta marginación son notablemente mayores a las del grupo de muy baja marginación, destacando que en el caso de la desnutrición la tasa es 11 veces mayor, y en las enfermedades infecciosas intestinales, la tasa quintuplica la del grupo de muy baja marginación. Es, además manifiesta la existencia de una clara tendencia al incremento de las tasas de mortalidad en la medida que el grado de marginación social es mayor, con la excepción de la tuberculosis pulmonar. 
Tabla 1 México: indicadores de mortalidad y de recursos y servicios, calculados por cuartil, y Coeficiente de Gini, 2005

\begin{tabular}{|c|c|c|c|c|c|c|}
\hline Tasas de Mortalidad' & $T$ & II & III & IV & Razón N/ & $\begin{array}{l}\text { Coef. } \\
\text { Gini }\end{array}$ \\
\hline Enf.Infecciosas Intestinales & 2,62 & 2,92 & 3,57 & 6,05 & 2,31 & 0,30 \\
\hline Tubercubsis Pulmonar & 2,35 & 1,38 & 1,32 & 2,86 & 1,21 & 0,41 \\
\hline Homicidios & 7,36 & 12,65 & 8,32 & 8,81 & 1,20 & 0,30 \\
\hline Mortalidad Materna & 45,27 & 69,45 & 53,45 & 78,93 & 1,74 & 0,18 \\
\hline Mortalidad Infantil & 13,69 & 15,58 & 18,31 & 21,15 & 1,55 & 0,11 \\
\hline Desnutrición & 6,30 & 6,71 & 9,45 & 10,48 & 1,66 & 0.21 \\
\hline Anemia Nutricional & 4,99 & 6,40 & 10,54 & 26,67 & 5,33 & 0,44 \\
\hline Ind. Recursos y Servicios & & & & & Razón ИV & \\
\hline$\%$ Partos Atendidos & 92,73 & 64,76 & 78,28 & 63,70 & 1,46 & 0,14 \\
\hline Gasto Pub Salud perépita & 3478,82 & 1717,42 & 2006,65 & 1659,67 & 2,10 & 0.25 \\
\hline Médicos por 100,000 hab & 173,90 & 91,50 & 100,63 & 78,31 & 2,22 & 0.27 \\
\hline Enfermeras por 100,000 hab & 230,38 & 124,13 & 122,89 & 97,50 & 2,36 & 0.29 \\
\hline Espec. Por Med General & 1,69 & 1,27 & 0,88 & 0,78 & 2,17 & 0.21 \\
\hline \% Población Derechohabiente & 59.93 & 49.63 & 45.81 & 32,96 & 1.79 & 0,17 \\
\hline
\end{tabular}

Por otra parte, esta tabla muestra algunas características socioeconómicas según categorías de marginación analizadas; de tal modo, se pone de manifiesto que la población residente en los municipios de muy alta marginación es eminentemente rural, en su gran mayoría la población ocupada recibe salarios bajos, tiene un alto grado de analfabetismo, y cerca de la mitad de las viviendas no tiene agua corriente en su interior, en franco contraste con las características de la población residente en los municipios de muy baja marginación. Casi la mitad de los municipios del país pueden ser considerados de alta y muy alta marginación, y en ellos vive alrededor del $17 \%$ de los mexicanos.

Tabla 2. México: Tasas de mortalidad (por 100 000) e indicadores socioeconómicos por municipios, agrupados según grado de marginación, 2005

\begin{tabular}{|c|c|c|c|c|c|c|}
\hline Tases de Morteibled & muy baja & beja & media & alts & muy slta & $\begin{array}{l}\text { Razon } \\
\text { maimb }\end{array}$ \\
\hline $\begin{array}{l}\text { End. Infecciosas } \\
\text { Imlestinales }\end{array}$ & 3,51 & 4,57 & 6,14 & 9,60 & 18,56 & 5,30 \\
\hline Tuberculosis Pumonar & 1,77 & 1,70 & 222 & 2,95 & 4,47 & 2,52 \\
\hline Dessnutriciôn & 6.71 & 10.38 & 12.37 & 13,06 & 7723 & 11.52 \\
\hline Anemias Nutricionales & 6.40 & 10,61 & 12,77 & 15,80 & 18,67 & 2,92 \\
\hline Homididos & 8,71 & B,95 & 8.87 & 11,18 & 16,68 & 1,92 \\
\hline Mortaldad Matema & 41.24 & 44,18 & 50,19 & 64,90 & 91,31 & 2,21 \\
\hline \multicolumn{7}{|l|}{ Indic. Socioeconómicess } \\
\hline Pociacion total & 5932212 & 15175714 & $11717 \$ 68$ & 12572021 & 4455535 & \\
\hline $\begin{array}{l}\text { Porcantaje de Pcb } \\
\text { que gana < } 2 \text { Sal. Minimos }\end{array}$ & 32,96 & 51,09 & 62,60 & 72,80 & 62,58 & \\
\hline $\begin{array}{l}\text { Porcentaje de Pob. } \\
\text { analtabeta > } 14 \text { atos }\end{array}$ & 3,60 & 8,46 & 12.88 & 19.76 & 34,50 & \\
\hline $\begin{array}{l}\text { Porcantsje de viviendss } \\
\text { sin mgua entubsa. }\end{array}$ & 3,30 & 9,58 & 14,17 & 25,80 & 42,94 & \\
\hline Nûmero de municipios & 279 & 423 & 501 & 896 & 365 & \\
\hline
\end{tabular}


Finalmente, en la Tabla 3 se presenta la información relativa al índice de inequidades en salud (INIQUIS) calculado para 2005, analizando tanto las tasas de mortalidad por causas relacionadas con el embarazo, parto y los primeros años de vida del niño como las tasas de mortalidad por causas potencialmente evitables. En ambos casos, el indicador más representativo (o "inicial”) seleccionado fue la tasa de mortalidad infantil. Los resultados revelan varios aspectos de interés.

Tabla 3. México: Índice de Inequidad en Salud por Estados: Indicadores de Mortalidad relacionada con el embarazo, parto y primera infancia* y de mortalidad potencialmente evitable ${ }^{\star *}, 2005$

\begin{tabular}{|c|c|c|c|}
\hline $\begin{array}{l}\text { Mort. R elacionada con el } \\
\text { embarazo, parto y primera } \\
\text { infanda }\end{array}$ & INIQUIS & Mort. Evitable & INIQUIS \\
\hline Coahuila & 0,056 & Aguascalentes & 0,114 \\
\hline Nuevo León & 0.081 & Nuevo Leb́n & 0,174 \\
\hline Tamaulipas & 0,157 & BCs & 0,197 \\
\hline Baja California & 0,192 & Distrito Federal & 0,204 \\
\hline Sonora & 0,195 & Zacatecas & 0,213 \\
\hline BCS & 02,208 & Guanajuato & 0,227 \\
\hline Chihuahua & 0.222 & Coahuila & 0,229 \\
\hline Distrito Federal & 0.233 & Jalisco & 0,240 \\
\hline Colima & 0249 & Quintana RoD & 0,241 \\
\hline Sinaloa & 0260 & Colima & 0,253 \\
\hline Morelos & 0.286 & Chihuahua & 0,272 \\
\hline Aguascalientes & 0.287 & Michoacan & 0,274 \\
\hline Nayarit & 02291 & Sonora & 0,297 \\
\hline Jalisco & 0.324 & Thaxcala & 0,312 \\
\hline Durango & 0,333 & Campeche & 0,318 \\
\hline Campeche & 0,368 & Tarnaulipas & 0,319 \\
\hline Quintana Roo & 0,380 & San Luis Potosí & 0,327 \\
\hline Tlaxcala & 0,386 & Yucatán & 0,337 \\
\hline Michoacân & 0,396 & Querétaro & 0,340 \\
\hline Zacatecas & 0.433 & Marelos & 0,342 \\
\hline Mb́xico & 0.452 & Sinaloa & 0,368 \\
\hline Querétaro & 0.456 & Hidalgo & 0,373 \\
\hline Hidalgo & 0,476 & Durango & 0,380 \\
\hline Guanajuato & 0.494 & Nayarit & 0,400 \\
\hline Yucatán & 0.495 & México & 0,409 \\
\hline Tabesco & 0,529 & Tabasco & 0,485 \\
\hline Veracruz & 0,536 & Baja Cal ifornia & 0,490 \\
\hline San Luis Potosi & 0,555 & Puebla & 0,500 \\
\hline Guertero & 0.627 & Veracruz & 0,545 \\
\hline Puebla & 0.635 & Chiapas & 0,583 \\
\hline Oaxaca & 0,796 & Guerrero & 0,640 \\
\hline Chiapas & 0.960 & Oaxaca & 0.704 \\
\hline
\end{tabular}


En primer lugar, en ambos análisis los estados que exhiben los más altos INIQUIS (Chiapas, Oaxaca, Guerrero, Puebla, Veracruz) se ubican en el Cuartil IV, aquel que agrupa a los estados de mayor grado de marginación.

De igual modo, los estados con los índices más bajos se ubican predominantemente en el Cuartil I, el cual concentra los estados menos marginados. A su vez, los resultados indican que siete de los 32 estados en cuanto a la mortalidad relacionada con el embarazo, parto y primera infancia, y cinco en lo referido a la mortalidad por causas potencialmente evitables, presentan una situación de salud claramente peor que la media del país, pues presentan valores superiores a 0,50, destacando el caso de Chiapas, que presenta un índice de 0,96 -una inequidad extrema- en lo concerniente a la mortalidad relacionada con la madre y el niño. Por el contrario, Nuevo León es el estado que tiene una mejor situación si se valoran ambos análisis -aparece en los dos en segundo lugar- y solo nueve estados pudieran considerase que presentan una situación buena en cada caso, dado que alcanzan valores menores a 0,25 (el Distrito Federal, Coahuila y Baja California Sur, además de Nuevo León, repiten su buena posición en ambos análisis)

\section{DISCUSIÓN}

El presente estudio puede tener algunas limitantes, referidas por un lado a la recolección de datos de mortalidad, y por otra, a los indicadores utilizados. En el primer caso, el bajo subregistro en la mortalidad reportado por la OPS para el período 2000-2005 (0,8 \%) (17) sin dudas avala la consistencia de los datos utilizados; sin embargo, es un hecho cierto que el nivel de subregistro de la mortalidad puede estar ligado, en sí mismo, a las desigualdades socioeconómicas, y por lo tanto, puede variar entre estados y municipios -siendo generalmente más elevado en las regiones más marginadas-, lo que pudiera reflejarse en una subestimación de las tasas observadas para estados y municipios con alta marginación.

Por otra parte, un índice como el INIQUIS -que aunque ha sido utilizado en distintos países latinoamericanos no ha sido validado aún en distintas condiciones (15)- tiene como atractivo fundamental ser un indicador único; sin embargo, al sintetizar se pierde la capacidad de indicar cuáles son los aspectos sobre los que hay que definir y formular programas específicos para mejorar la salud. Obviamente, la selección de distintos indicadores de "inicio" puede producir resultados divergentes. No obstante, los resultados hallados en el estudio parecen suficientemente consistentes entre sí, lo que avala la robustez del método empleado. 
A su vez, debe tenerse presente que la razón de tasas entre cuartiles extremos deja de lado los posibles cambios en las condiciones de los grupos intermedios, y solo resalta las diferencias entre posiciones sociales claramente opuestas. No obstante, se aprecia en términos generales una tendencia al incremento de las tasas de mortalidad (o a la disminución de la disponibilidad de recursos y servicios) en la medida que aumenta el grado de marginación, lo que sugiere que la marginación social desempeña un notable papel para explicar las desigualdades sanitarias en el plano socio-espacial.

En todo caso, estos aspectos antes mencionados no parecen cuestionar los hallazgos del estudio, que ponen de manifiesto la notoria inequidad sanitaria que aún existe en el país - más notable en la mortalidad potencialmente evitable y en aquella relacionada con el embarazo, el parto y la primera infancia-, inequidad asociada a las evidentes disparidades socioeconómicas subyacentes entre las distintas regiones de México y que nos remiten sobre todo a lo injusta e innecesaria de estas desigualdades, que excluyen a importantes conjuntos poblacionales de opciones adecuadas para la satisfacción de sus necesidades básicas.

Como señala la OPS, “'los niveles de pobreza en México siguen siendo elevados para un país con ingresos altos, miembro desde 1994 de la Organización para la Cooperación y el Desarrollo Económicos (OCDE)" y que se ubica entre las más importantes economías del mundo (17); junto a estos niveles de pobreza, las brechas, las inequidades y las diferencias de oportunidades entre la población son características del México actual, que se han mantenido a lo largo de las últimas décadas (8).

El análisis por entidad federativa, agrupadas en cuartiles, permite caracterizar a un conjunto de estados -los ubicados en el Cuartil IV, geográficamente situados al sur del país- que reúne a la mitad de la población rural de México y al $70 \%$ de su población indígena, con una quinta parte de las viviendas sin agua corriente en su interior y $15 \%$ de personas de 15 años y más analfabetas.

Lo anterior significa que en estos estados, grandes grupos poblacionales han quedado excluidos de la dinámica social, sin alternativas reales para satisfacer sus necesidades esenciales, y por ende, esto se traduce en un mayor riesgo para la salud, especialmente cuando se trata de muertes por causas potencialmente evitables; para algunos autores, las condiciones socioeconómicas están más asociadas con la mortalidad prevenible que con las causas de muerte difícilmente evitables debido a que el conocimiento que existe para prevenir oportunamente un problema de salud puede ser aprovechado mejor por aquellos que 
viven en una situación socialmente ventajosa y disponen de más recursos económicos y mejor acceso a los servicios de salud (18).

Con el análisis del INIQUIS, que otorga la mayor puntuación a varios de los estados agrupados en este cuartil, se confirma esta situación de desventaja sanitaria. A su vez, estos estados son los que tradicionalmente han recibido menos apoyos y recursos en el sector salud (8), donde menos gasto público se destina a este rubro y por lo tanto, hay mayor escasez de recursos humanos y materiales para resolver los problemas de salud: así, a la inequidad en los indicadores de mortalidad debe agregarse la inequidad en el acceso a los servicios de salud.

Si bien un análisis de los estados que ocupan los puestos extremos en cuanto a marginación social revelaría distancias aún mayores en los indicadores sanitarios -tanto en los de resultados como en los de recursos y servicios-, los resultados demuestran que no solo son Guerrero, Chiapas y Oaxaca los estados con peores indicadores de salud: también Puebla, Veracruz o Hidalgo, por citar solo algunos estados, presentan características similares, y deben ser objeto de especial atención.

A los aspectos previamente descritos se suma una arista demográfica: en estos ocho estados agrupados en el Cuartil IV hay en la actualidad -y no parece que esto se modifique en el futuro cercano- más niños menores de 15 años, más nacimientos (y una mayor tasa global de fecundidad), más nacimientos provenientes de madres adolescentes ( $\mathrm{y}$ una mayor tasa de fecundidad adolescente) y un mayor número de ancianos que en cualquier otro cuartil (19); o sea, un mayor volumen de niños y ancianos están expuestos a los mayores riesgos de mortalidad potencialmente prevenible, no por desigualdades inevitables (como aquellas debidas a las diferentes características biológicas o genéticas), sino por desigualdades injustas e innecesarias asociadas al hecho de vivir en un contexto geográfico de alta marginación (20). En este escenario, cobran especial relevancia las políticas sanitarias encaminadas a mejorar las condiciones de salud de la niñez y a reducir las desigualdades en salud en la infancia-, pues estas tienen un mayor impacto a largo plazo, debido a la influencia que tienen las condiciones de salud en los primeros años de vida en la salud adulta (21).

En tal sentido, el análisis por grado de marginación a nivel municipal parece confirmar lo expuesto en párrafos previos: 17 \% de la población mexicana vive en municipios de alta o muy alta marginación; son predominantemente rurales, indígenas, con bajos ingresos y bajos niveles educativos, y están expuestos a riesgos de morir por causas que en gran medida se pudieran evitar muy superio- 
res a las de los municipios con bajos niveles de marginación. Basta destacar que la mortalidad por desnutrición en el grupo de municipios con muy alta marginación más que decuplica la tasa observada en el grupo de municipios con muy baja marginación.

Si se asume que la exclusión social es resultado de la convergencia de procesos estructurales - factores financieros, económicos, políticos, tales como el desempleo o el subempleo, la falta de apoyos a actividades económicas, la desprotección social, o la falta de procesos realmente democráticos, por ejemplo- y el entorno social- como las transformaciones demográficas y los procesos migratorios, o la fragilidad de las redes sociales actuales, entre otros-(4), para modificar esta situación inequitativa en términos sanitarios también se necesitan profundos cambios estructurales que impulsen el desarrollo social, y permitan reducir las desventajas injustas a las que están expuestos importantes núcleos poblacionales del país.

En este contexto, revertir la inequidad implica generar empleos dignos (la carencia de un empleo formal es en si misma un factor excluyente de la seguridad social); apoyar decididamente al campo mexicano y a los campesinos -que no es lo mismo que apoyar a los grandes productores-; luchar contra la discriminación de género y étnica; fortalecer los sistemas de salud y de seguridad social - y no solo crear alternativas como el seguro popular para la población que no cuenta con seguridad social y que por lo tanto (siendo la que carece de empleo estable) debe pagar por la atención médica-; democratizar la vida política y lograr una mayor participación comunitaria y redistribuir, desde el gobierno federal, la riqueza de forma verdaderamente equitativa, otorgando más recursos a los estados y municipios que más necesidades de salud tienen y rompiendo inercias y prácticas burocráticas en la asignación de recursos humanos y materiales

\section{REFERENCIAS}

1. De Ferranti D, Perry G, Ferreira F, Walton M. Desigualdad en América Latina y el Caribe: ¿Ruptura con la historia? Washington D.C.: Banco Mundial, 2003: pp. 3-40.

2. Secretaría de Desarrollo Social. Pobreza y Marginación Social 2005. En: Doble Carta. Seminario de Información Socioeconómica, SEDESOL, México DF, 2006.

3. Consejo Nacional de Población. Indicadores socioeconómicos e índice de marginación municipal, 1990. Primer informe técnico del proyecto Desigualdad regional y marginación municipal en México. México, Consejo Nacional de Población. 1993

4. Bel AC. Exclusión Social: origen y características. En Curso: "Formación específica en Compensación Educativa e Intercultural paraAgentes Educativos". Murcia, Enero de 2002. [Internet]. Disponible en: http://stepv.intersindical.org/enxarxats/nee/CE_exclusio.pdf Consultado Septiembre 28, 2007. 
5. Starfield B. State of the Art in Research on Equity in Health. Journal of Health Politics, Policy and Law, 2006; 31(1): 11-32

6. Engels F. La situación de la clase obrera en Inglaterra (1845). Editorial Ciencias Sociales, La Habana 1972.

7. International Society for Equity in Health (ISEQH). [Internet]. Disponible en: http://www.isegh.org/ workdef_sp.htm Consultado Septiembre 27, 2005.

8. Zurita B, Lozano R, Ramírez T, Torres JL. Desigualdad e Inequidad en Salud, En: Knaul F, Nigenda G, eds "Caleidoscopio de la Salud", México, Fundación Méxicana para la Salud; 2003. pp 29-39.

9. Instituto Nacional de Estadística, Geografía e Informática (INEGI) II Conteo de Población y Vivienda 2005. INEGI, Aguascalientes; 2006.

10. United Nations Children's Fund (UNICEF) The State of the World's Children 2007. UNICEF, New York, NY; 2006

11. United Nations Development Programme (UNDP). Human Development Report 2006. New York, NY; Palgrave MacMillan; 2006

11. Sistema Nacional de Información en Salud de la Secretaría de Salud (SINAIS). Base de datos de Mortalidad 1979-2006, [Internet]. Disponible en: http://sinais.salud.gob.mx/basesdedatos/ defunciones.html Consultado Septiembre 26, 2007

12. Consejo Nacional de Población (CONAPO) Proyecciones de la población de México, 2000-2050. CONAPO, México DF; 2002.

13. Consejo Nacional de Población (CONAPO) Proyecciones de la población de México, 2005-2050. CONAPO, México DF, 2006. [Internet]. Disponible en: http://www.conapo.gob.mx/00cifras/5.htm Consultado Septiembre 22, 2007

14. Xunta de Galicia. OPS. Epidat. Programa para análisis epidemiológico de datos tabulados, versión 3.1., Washington, DC; enero 2006.

15. Consejo Nacional de Población (CONAPO). Índices de Marginación a nivel localidad 2005. CONAPO, México DF, 2007. [Internet]. Disponible en: http://www.conapo.gob.mx/publicaciones/indice2005xloc.htm Consultado Septiembre 26, 2007

16. Organización Panamericana de la Salud. México en OPS: La Salud en las Américas 2007, Washington; 2007. p. 524-544

17. Phelan, J. C., B. G. Link, A. Diez-Roux, I. Kawachi, and B. Levin. Fundamental Causes of Social Inequalities in Mortality: A Test of the Theory. Journal of Health and Social Behavior, 2004; 45: 265-285.

18. González-Pérez GJ, Vega-López MG, Muñoz de la Torre A, Cabrera-Pivaral CE, Romero-Valle S. Demografía, Equidad y Salud en la Infancia: Realidades y Desafíos para Latinoamérica En: Vega-López MG, González-Pérez GJ (coord) Infancia, Sociedad y Salud. Guadalajara, México: Ediciones de la Noche, Universidad de Guadalajara y OPS; 2006. p. 339-359.

19. Whitehead M . The concepts and principles of equity and health. Int J Health Serv, 1992; 11 (3): 429-445.

20. Davey-Smith G, Lynch J. Commentary: Social Capital, Social Epidemiology and Disease Aetiology. International Journal of Epidemiology. 2004; 33:691-700. 